\title{
F $\begin{array}{ll}\text { Research Square } & \text { They should not be considered conclusive, used to inform clinical practice, } \\ \text { or referenced by the media as validated information. }\end{array}$
}

\section{Immunity Improvement and Gut Microbiota Remodeling of Mice by Wheat Germ Globulin}

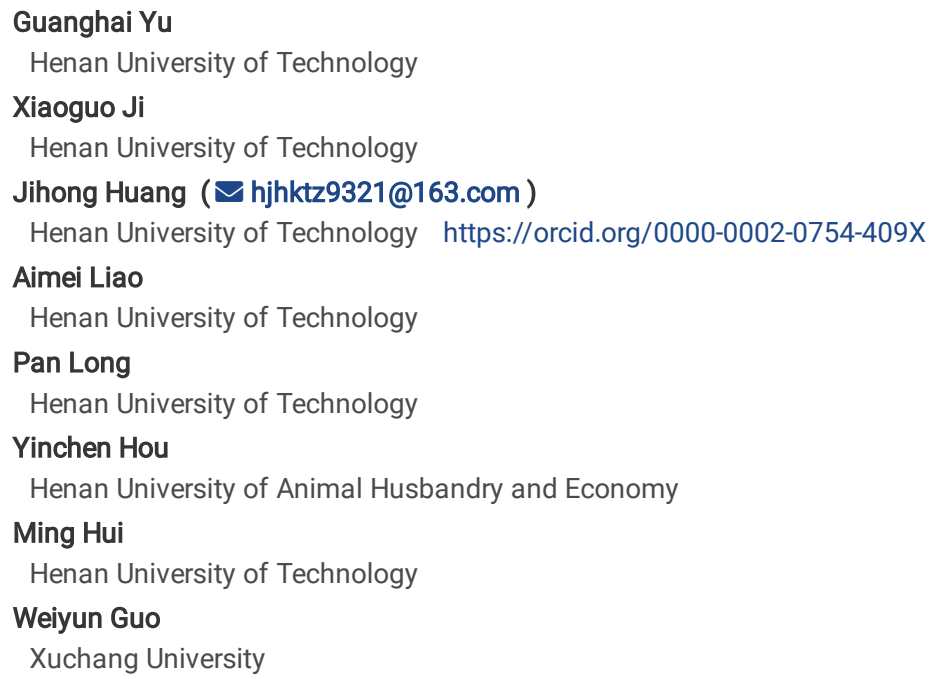

Version of Record: A version of this preprint was published at World Journal of Microbiology and Biotechnology on March 18th, 2021. See the published version at https://doi.org/10.1007/s11274-021-03034-1. 


\begin{abstract}
The wheat germ protein (WG) and it's proteolytic peptide have a variety of biological activities. Our previous work showed that WG could improve immunity of the immunosuppressive mice established by cyclophosphamide. However, in the healthy condition and normal diet, as a supplementary food, the effects of immunity improvement and gut microbiota remodeling by the wheat germ globulin has not been studied yet. Here, we reported that WG could improve the immunity and remodel the gut microbiota of the mice, as a safe functional supplementary food for the first time. The increase of interleukin- 6 (IL-6) and the decrease of tumor necrosis factor a (TNF-a) and interleukin-10 (IL-10) indicated that WG could enhance the levels of activated T cells and monocytes and antiinflammatory ability, meanwhile, the significant increase of immunoglobin $\mathrm{G}(\mathrm{lgG})$ and the notable decrease of the immunoglobin $\mathrm{M}$ ( $\mathrm{lgM}$ ) and immunoglobin A (IgA) illustrated that WG could improve immunity by promoting the differentiation and maturation process of B cells, compared with the NC group. 16S rRNA sequencing showed WG could remodel the gut microbiota. At the phylum level, the Bacteroidetes were reduced and Firmicutes were increased in WG group, compared with NC group. At the genus level, the SCFA producing genera of unclassified_f_Lachnospiraceae, Blautia and especially the Roseburia (increased more than threefold) increased notably. Further, the level changes of cytokines and immunoglobulins were associated with the gut microbiota. This work showed that WG could improve immunity and has potential application value as an immune-enhancing functional food.
\end{abstract}

\title{
Introduction
}

There are more than 500 species of microbes colonized in the intestine and the gut microbiota are heavily involved in the modulating of immunity, inflammation, metabolism of carbohydrates and fats, and in gut-brain neural circuits, which maintains a complex dynamic balance to effectively resist the invasion of pathogens (Dai et al. 2020; Hegazy et al.). Intestinal microbes participate in and regulate the host's immune response through bile acids, lipoproteins, lipopolysaccharides, unsaturated fatty acids and other metabolites to form an intestinal immune protective barrier (Anh et al. 2019; Castellanos and Longman 2020 ; Sanna et al. 2019; Vitetta et al. 2018 ; Wan et al. 2019). Short-chain fatty acids (SCFAs) derived from metabolism of the gut microbiota are crucial for intestinal health and participate in the maintenance of other important physiological functions of the body (Chang et al. 2020; Shuo et al. 2018). It was reported that the SCFAs, in particular butyrate, exerted important immuno-modulatory functions (Martin-Gallausiaux et al. 2020; Stilling et al. 2018).

The wheat germ is an important by-product of the flour milling industry. After oil extraction, the germ meal has high protein content (over $30 \%$ ), with globulin about $15 \%$ of total protein. And the wheat germ protein presents a well-balanced amino acid profile, which is a potential supplementary food with high nutritional value (Brandolini and Hidalgo 2012). The wheat germ protein and it's proteolytic peptide have a variety of biological activities. Wheat germ proteolytic peptide WG-P exerted an effective antioxidant action by across the intestinal epithelium (Zhang et al. 2019a). The Wheat Germ Globulin proteolytic peptide ECFSTA exerted immunomodulatory effects (Wu et al. 2017). Our previous work showed that wheat germ globulin (WG) could improve the immune function of immunosuppressive mice established by intraperitoneal injection of cyclophosphamide (Ji et al. 2018). However, in the healthy condition and normal diet, whether WG as a functional supplementary food could also improve immunity and whether its immune-promoting effect is related to the improvement of intestinal microbiota has not been studied yet.

In this work, the safety of WG as a functional supplementary food was verified. Further, the effects of immunity improvement and gut microbiota remodeling by the wheat germ globulin were investigated.

\section{Materials And Methods}

\section{Animal Groups and protein Administration}

$48 \mathrm{BALB} / \mathrm{c}$ male mice (age of 6-8 weeks,weight of 18-22 g), purchased from the Experimental Animal Center of Zhengzhou University, were fed under specific pathogen-free conditions at a temperature of $25^{\circ} \mathrm{Cand}$ humidity of $60 \%$. The mice were fed with the standard laboratory diet according to AIN-93G standards (Ji et al. 2018). Then, the 48 mice were divided averagely into four groups at random and fed different protein samples (with the same calorie levels), in addition to the standard diet, as shown in Table 1.

Table 1

Animal model grouping

\begin{tabular}{|ll|}
\hline Group & Intragastric administration (30 days) \\
\hline Normal controlgroup (NC) & Saline \\
\hline Wheat germ globulin group (WG) & $100 \mathrm{mg} \mathrm{WG/kg} \mathrm{BW/day}$ \\
\hline Egg white protein group (EG) & $100 \mathrm{mg} \mathrm{EG} \mathrm{/kg} \mathrm{BW/day}$ \\
\hline Whey Protein group (RG) & $100 \mathrm{mg} \mathrm{DG} \mathrm{/kg} \mathrm{BW/day}$ \\
\hline Groups division: NC, normal control group; WG, wheat germ globulin group; EG, Egg white protein group;RG: Whey Protein group. \\
\hline
\end{tabular}

\section{Hematological parameters and hematological biochemical parameters Analysis}

The blood supernatant were prepared and used to detect blood indexes using the automatic biochemical analyzer Coulter-JT (Beckman Coulter Co., Ltd. USA). Inflammatory Factors and Immunoglobulins Analysis by ELISA

Peripheral blood samples were collected from the mice eyeball, and were kept at room temperature for 2 hours. Then, the samples were centrifuged at $2000 \mathrm{~g}$ for 20 minutes. The serum was collected and stored at $-20^{\circ} \mathrm{C}$ without repeated freezing and thawing. The levels of IL-IB, IL-6, IL-10, TNF-a, IFN-ץ, IgG, IgA and 
IgM of each sample were determined according to the instructions of the ELISA kit (NeoBioScience Co., Ltd. Beijing,China).

\section{Acquisition of intestinal contents and genomic DNA extraction}

The ileocecal areas of the mice were cut and washed with PBS. Then, the precipitate was collected by centrifugation, which was freezed in liquid nitrogen for $0.5 \mathrm{~h}$ and transfered to $-80^{\circ} \mathrm{C}$ for storage. Microbial community genomic DNA was extracted by the E.Z.N.A. (B soil DNA Kit (Omega Bio-tek, Norcross, GA, U.S.) according to manufacturer's instructions. The hypervariable region V3-V4 of the bacterial 16S rRNA genes were amplified with primer pairs $338 \mathrm{~F}$ and $806 \mathrm{R}$ (338F: 5'-ACTCCTACGGGAGGCAGCAG-3';806R: 5'-GGACTACHVGGGTWTCTAAT-3') by an ABI GeneAmp® 9700 PCR thermocycler (ABI, CA, USA) and performed in triplicate. The PCR product was extracted from $2 \%$ agarose gel and purified using the AxyPrep DNA Gel Extraction Kit (Axygen Biosciences, Union City, CA, USA) according to manufacturer's instructions and quantified using Quantus ${ }^{\mathrm{TM}}$ Fluorometer (Promega, USA).

\section{S rRNA gene sequencing and processing of sequencing data}

Purified amplicons were pooled in equimolar and paired-end sequenced $(2 \times 300)$ on an Illumina MiSeq platform (Illumina, San Diego, USA) according to the standard protocols by Majorbio Bio-Pharm Technology Co. Ltd. (Shanghai, China). The raw sequences of 16S rRNA gene sequencing have been deposited in the NCBI Sequence Read Archive (SRA) database under Accession Number: (SAMN17192475, SAMN17192476,SAMN17192477,

SAMN17192478,SAMN17192479,SAMN17192480, SAMN17192481, SAMN17192482, SAMN17192483, SAMN17192484, SAMN17192485, AMN17192486, SAMN17192487, SAMN17192488, SAMN17192489). The raw sequencing reads were demultiplexed, quality-filtered by Trimmomatic and merged by FLASH with the following criteria: (1) the $300 \mathrm{bp}$ reads were truncated at any site receiving an average quality score of $<20$ over a 50 bp sliding window, and the truncated reads shorter than $50 \mathrm{bp}$ were discarded, reads containing ambiguous characters were also discarded; (2) only overlapping sequences longer than $10 \mathrm{bp}$ were assembled according to their overlapped sequence. The maximum mismatch ratio of overlap region is 0.2 . Reads that could not be assembled were discarded; (3) Samples were distinguished according to the barcode and primers, and the sequence direction was adjusted, exact barcode matching, 2 nucleotide mismatch in primer matching. Operational taxonomic units (OTUs) with $97 \%$ similarity cutoff were clustered using UPARSE (version 7.1, http://drive5.com/uparse/), and chimeric sequences were identified and removed. The taxonomy of each OTU representative sequence was analyzed by RDP Classifier (http://rdp.cme.msu.edu/) against the Silva database (SSU128) using confidence threshold of 0.7.

\section{Statistical analysis}

The experimental data were analyzed with software SPSS version 19.0 for Windows. Analysis of variance was conducted by Duncan's multiple range tests to determine significant differences between the samples. The statistical significance was set at $P<0.01$ to indicate very significant differences, $P<0.05$ to indicate significant difference, and $P>0.05$ to indicate absence of significant differences.

\section{Results}

\section{Wheat germ globulin could be used as a long-term safe supplementary dietary protein}

As shown in Table 2, there were no significant differences in the hematological parameters among the four groups on the whole, and they were all within the normal range, although there were some differences in some indexes, such as leukocytes and some indexes of platelet. Similarly, there were also no notable differences in the hematological biochemical parameters among the WG group and the NC, RG, and EG groups on the whole, and they were all within the normal range, although there were some differences in some indexes ( Table 3). Compared with NC, the high-density lipoprotein index of the WG group increased while the low-density lipoprotein index decreased.

Table 2

Comparison of hematological parameters on different treatment group mice

\begin{tabular}{|lllll|}
\hline Item & NC-GROUP & WG-GROUP & EG-GROUP & RG-GROUP \\
\hline Leukocytes $\left(\times 10^{9} / \mathrm{L}\right)$ & $3.80 \pm 0.48$ & $8.68 \pm 0.55$ & $5.72 \pm 0.21$ & $7.88 \pm 0.35$ \\
\hline Red blood cell $\left(\times 10^{9} / \mathrm{L}\right)$ & $7.32 \pm 0.02$ & $8.12 \pm 0.13$ & $7.17 \pm 0.21$ & $8.59 \pm 0.14$ \\
\hline Hematocrit $(\%)$ & $25.26 \pm 4.09$ & $37.40 \pm 1.05$ & $33.80 \pm 0.70$ & $39.10 \pm 2.28$ \\
\hline Mean hematocrit(fv) & $45.35 \pm 0.15$ & $46.07 \pm 1.43$ & $47.53 \pm 1.37$ & $45.43 \pm 1.97$ \\
\hline Red blood cell distribution width(\%) & $22.77 \pm 5.47$ & $17.00 \pm 2.40$ & $15.47 \pm 1.02$ & $14.04 \pm 0.80$ \\
\hline Red blood cell distrution width $\mathrm{SD}(\mathrm{fL})$ & $36.00 \pm 4.00$ & $33.00 \pm 5.03$ & $31.00 \pm 3.00$ & $27.33 \pm 0.66$ \\
\hline Platelet count( $\left.\times 10^{9} / \mathrm{L}\right)$ & $667.00 \pm 9.00$ & $941.67 \pm 7.49$ & $1309.00 \pm 9.00$ & $695.50 \pm 67.50$ \\
\hline Plateletcrit $(\%)$ & $0.72 \pm 0.22$ & $0.54 \pm 0.05$ & $0.67 \pm 0.04$ & $0.41 \pm 0.04$ \\
\hline Platelet volume(fL) & $8.05 \pm 0.35$ & $5.70 \pm 0.17$ & $5.47 \pm 0.03$ & $5.33 \pm 0.07$ \\
\hline Platelet distribution width $(\%)$ & $15.07 \pm 0.20$ & $14.70 \pm 0.15$ & $14.47 \pm 0.12$ & $14.43 \pm 0.09$ \\
\hline Hemoglobin(g/L) & $164.50 \pm 0.50$ & $136.67 \pm 1.45$ & $108.00 \pm 1.00$ & $130.00 \pm 8.18$ \\
\hline Mean corpuscular hemoglobin(pg) & $19.25 \pm 2.25$ & $16.80 \pm 0.25$ & $15.17 \pm 0.19$ & $15.10 \pm 0.70$ \\
\hline Mean corpuscular hemoglobin concentration(g/L) & $424.50 \pm 5.05$ & $379.50 \pm 0.50$ & $319.00 \pm 5.29$ & $332.33 \pm 1.86$ \\
\hline
\end{tabular}


Table 3

Comparison of hematological biochemical parameters on different treatment group model mice

\begin{tabular}{|lllll|}
\hline Item & NC-GROUP & WG-GROUP & EG-GROUP & RG-GROUP \\
\hline Alanine aminotransferase (ALT) (U/L) & $32.90 \pm 2.40$ & $31.10 \pm 1.70$ & $33.40 \pm 0.36$ & $31.03 \pm 1.33$ \\
\hline Aspartate aminotransferase(AST) & $147.83 \pm 5.65$ & $158.25 \pm 6.75$ & $158.37 \pm 8.99$ & $139.67 \pm 7.19$ \\
$(\mathrm{U} / \mathrm{L})$ & & & & \\
\hline AST/ALT & $3.78 \pm 1.02$ & $5.12 \pm 0.70$ & $4.73 \pm 0.90$ & $4.52 \pm 0.59$ \\
\hline Total protein(g/L) & $47.17 \pm 0.88$ & $47.23 \pm 1.14$ & $45.83 \pm 2.04$ & $47.43 \pm 1.44$ \\
Albumin(A) (g/L) & $28.57 \pm 0.58$ & $27.40 \pm 0.23$ & $27.50 \pm 1.70$ & $28.50 \pm 0.95$ \\
\hline Globulin (G)(g/L) & $18.60 \pm 0.35$ & $19.83 \pm 0.96$ & $19.50 \pm 1.20$ & $18.93 \pm 0.60$ \\
\hline A/G & $1.53 \pm 0.03$ & $1.40 \pm 0.58$ & $1.40 \pm 0.00$ & $1.53 \pm 0.03$ \\
\hline Carbonyldiamide(mmol/L) & $10.03 \pm 0.16$ & $10.55 \pm 0.14$ & $7.54 \pm 0.21$ & $6.80 \pm 0.38$ \\
\hline High density protein(mmol/L) & $1.56 \pm 0.02$ & $1.78 \pm 0.14$ & $1.83 \pm 0.08$ & $1.79 \pm 0.11$ \\
\hline Low density protein(mmol/L) & $0.20 \pm 0.02$ & $0.18 \pm 0.02$ & $0.28 \pm 0.03$ & $0.20 \pm 0.01$ \\
\hline
\end{tabular}

\section{Wheat Germ Globulin could improve Immunity of mice}

The levels of cytokines involved in inflammation and immunoglobulins in the peripheral blood of four groups of NC, WG, EG, and RG were compared, in order to evaluate the effects of wheat germ globulin in immunity improvement (Fig. 1). There were no significant changes of the levels of IFN- $\gamma$ and IL-1 $\beta$ for WG and RG groups, except for EG, compared with the NC group (Fig. 1A and Fig. 1C). The change trends of levels of IL-6, TNF-aand IL-10 for WG, EG and RG groups were the same, compared with the NC group. The levels of IL- 6 of the WG, EG and RG groups increased significantly, with the WG group the most ( increased 93.2\%), while the levels of TNF-a and IL-10 of the WG, EG and RG groups decreased slightly, compared with the NC group (Fig. 1B, Fig. 1D and Fig. 1E).

Further, the levels of IgG of WG, EG and RG groups were increased significantly by $17.8 \%, 5 \%$ and $13.4 \%$, respectively, compared with the NC group (Fig. $1 \mathrm{G}$ ). While the levels of IgM of WG, EG and RG groups were decreased significantly by $37.1 \%, 41.2 \%$ and $36.1 \%$, respectively, compared with the NC group (Fig. 1F). And the levels of IgA of WG and RG groups were decreased significantly by $28.8 \%$ and $21.5 \%$, respectively, except for EG, compared with the NC group (Fig. 1H).

Sampling depth, alpha diversity and the overall gut microbial community structure difference of the four groups samples of NC, WG, RG and EG

A total of 555,855 high-quality reads, with the average length of $435 \mathrm{bp}$, were obtained from 15 samples which were assigned to 466 0TUs (Phylum:9, Class:15, Order:20, Family:31, Genus:90 and Species:159), based on $\geq 97 \%$ sequence similarity (Supplementary Table 1, Table 2). The rarefaction curves showed that the sequencing data could cover the majority of bacterial diversity (Supplementary Fig. 1). There was no significant difference in alpha diversity among the four groups samples of NC, WG, RG and EG, which indicated that the regulation effect of WG, RG and EG on intestinal organisms were mainly by changing the community structures rather than increasing species abundances (Supplementary Fig. 2). As revealed by principal coordinate analysis (PCA) at operational taxonomic unit (OTU) level, the overall gut microbiota structure of the four groups samples of NC, WG, RG and EG were structurally different from each other and displayed a distinct separation pattern (Supplementary Fig. 3).

\section{Microbiota composition and difference analysis of the four groups samples of NC, WG, RG and EG}

At the phylum level, Firmicutes and Bacteroidetes were the most abundant in the four groups samples of NC, WG, RG and EG and together they make up more than $95 \%$ of the total, as shown in Fig. 2A. Compared with the NC group, the Bacteroidetes in the WG group were significantly reduced and Firmicutes were significantly increased, that is, the ratio of Bacteroidetes to Firmicutes (B/F ratio) was significantly reduced (Fig. 2B).

At the genus level, the microbiota compositions of the four groups of NC, WG, RG and EG were significantly different. The norank_f_Bacteroidales_S24-7, Lachnospiraceae_NK4A136, norank_f_Lachnospiraceae, Lactobacillus, unclassified_f_Lachnospiraceae, Roseburia and Alistipes were the most abundant in the four groups of NC, WG, RG and EG (Fig. 3A). Beneficial bacteria of Roseburia (NC: $2.27 \%, W G: 9.19 \%$, EG: 2.65\%, RG:2.37\%), capable of producing butyric acid, was remarkably increased in the WG group, compared with the groups of NC, EG and RG, although the P value was slightly greater than 0.05 ( 0.05556 ), as shown in Fig. 3B. The abundance of norank_f_Bacteroidales_S24-7 observably decreased in the WG group, while the abundance of unclassified_f_Lachnospiraceae both in groups of NC and WG was significantly higher than that in groups of EG and RG ( $p<0.05)$ (Fig. 3B). It was worth noting that the beneficial bacteria of Blautia was remarkably increased in the WG group, compared with the groups of NC, EG and RG ( $p<0.05$ ) (Fig. 3B).

Further, the microbiota composition difference between the groups of NC and WG was analyzed (Fig. 4). Beneficial bacteria of Roseburia (NC: $2.27 \%$,WG: $9.19 \%)$, capable of producing butyric acid, was remarkably increased in the WG group $(\mathrm{p}<0.05)$, while the abundance of norank_f_Bacteroidales_S24-7 observably decreased in the WG group $(p<0.05)$, compared with the group NC, as shown in Fig. 4.

\section{Correlation Analysis Between Gut Microbiota And Cytokines And Immunoglobulin}


To determine whether there were potential associations between the alteration of the gut microbiota and biochemical parameters of cytokines and immunoglobulin, we analyzed the correlation between the relative abundance of the most abundant 50 genera and the biochemical parameters by Spearman correlation analysis. As shown in Fig. 5, IL-6 was positively correlated with Anaeroplasma $(\mathrm{p}<0.05)$ and negatively correlated with Parasutterella ( $<<0.05)$ and Bifidobacterium $(\mathrm{p}<0.05)$. IL-10 showed positive correlation with Blautia $(\mathrm{p}<0.05)$, Bifidobacterium $(\mathrm{p}<0.05)$, Rikenella $(\mathrm{p}<0.05)$ and

norank_f_Clostridiales_vadinBB60_group $(p<0.05)$, while negative correlation with Ruminococcaceae_UCG-009 $(p<0.05)$. TNF-a was positively correlated with Ruminiclostridium_5 $(\mathrm{p}<0.05)$ and negatively correlated with Bacteroides $(\mathrm{p}<0.05)$.

As shown in Fig. 6, IgG showed positive correlation with Anaeroplasma $(\mathrm{p}<0.05)$, Anaerotruncus $(\mathrm{p}<0.05)$ and Ruminococcaceae_UCG-009( $\mathrm{p}<0.05)$, while negative correlation with norank_o_Mollicutes_RF9 $(p<0.05)$, Parasutterella $(p<0.05)$, unclassified_f_Ruminococcaceae $(p<0.05)$ and Bifidobacterium $(p<$ $0.01)$. While, IgM was positively correlated with Rikenella $(\mathrm{p}<0.05)$. The $\lg A$ was notably positively correlated with unclassified_f_Ruminococcaceae $(\mathrm{p}<0.01)$ and Ruminococcaceae_UCG-014 ( $p<0.05)$, while negatively correlated with Lachnospiraceae_UCG-006 $(p<0.05)$ and Anaeroplasma $(p<0.05)$. The above research results indicated that the alteration of the gut microbiota was one of the important reasons for the level changes of cytokines and immunoglobulin.

\section{Discussion}

There were no significant differences in the hematological parameters and hematological biochemical parameters among the WG group and the NC, RG, and EG groups on the whole and they were all within the normal range, which indicated that WG could be used as a long-term safe supplementary dietary protein. Further, the high-density lipoprotein index increased while the low-density lipoprotein index decreased, in WG group, compared with NC group, indicating that the high-protein diet supplemented with WG was beneficial for reducing blood lipids.

The levels of IL-6 increased significantly, with the WG group the most (increased 93.2\%), while the levels of TNF-a and IL-10 decreased slightly, in the groups of WG, EG and RG, compared with the NC group. IL-6 is mainly synthesized and secreted by activated T lymphocytes and peripheral blood mononuclear cells, and has a positive regulatory effect on immunity (Farsakoglu et al. 2019; Muhsin et al. 2018). While IL-10 has a negative regulatory effect on immunity (Matsumoto et al. 2009; Neumann et al. 2019). Thus, the increase of IL-6 levels and the decrease of the IL-10 levels indicated the improve of immunity by enhancing the levels of activated T cells and monocytes. TNF-a is an important pro-inflammatory cytokine, and the decrease of its level indicating the increase in the body's anti-inflammatory ability (Decourt et al. 2017; Kemanetzoglou and Andreadou 2017). The level of IgG increased significantly by $17.8 \%$, while the levels of IgM and IgA decreased significantly by $37.1 \%$ and $28.8 \%$, respectively, compared with the NC group. The change trends of levels of IgG in both the RG and EG groups were as same as the WG group, but with smaller change levels. The lgG is the main component of the antibodies in serum and body fluids, accounting for about $80 \%$ of the total serum immunoglobulins. Serum IgG can neutralize pathogens or toxins and activate the classical or alternative pathway of complement, forming a membrane attack complex (MAC) on the surface of the pathogen's membrane and turning on complement-dependent cytotoxicity (CDC) (Xie et al. 2020; Ząbczyńska et al. 2020). The differentiation and maturation process of B cells is divided into five stages: immature B cells (im-B), mature $B$ cells (ma-B), activated B cells (ac-B), memory B cell and plasma cells (Wang et al. 2020). The lgM was synthesized by im-B, ma-B and ac-B. And the memory B cell can synthesize both IgG and IgA, while plasma cells only synthesize and secrete $\lg$ G to mediate humoral immunity (Martin-Subero and Oakes 2018; Sigvardsson 2018). Therefore, the increased levels of high-affinity IgG and the decreased levels of low-affinity lgM and IgA indicated that WG were beneficial to promote the differentiation and maturation of B cells and improve humoral immunity. In summary, protein supplementation could improve the body's immunity and the effect of WG is better than that of EG and RG. Our previous research work found that WG could reduce the immunosuppression caused by cyclophosphamide by enhancing the levels of IL-2 and IL-4 and the ratio of CD4+/CD $8+$ and restoring Th1/Th2 imbalance (Ji et al. 2018). The results of this work could prove that WG could improve the body's immunity, from another perspective and has potential application value as an immuneenhancing functional food.

16S rRNA sequencing showed WG could remodel the gut microbiota. At the phylum level, the Bacteroidetes were reduced and Firmicutes were increased in WG group, compared with NC group. Although studies have shown that a lower B/F ratio is good for gut health (Xu et al. 2020), there have been cases where the opposite results have been found. There is growing consensus that the B/F ratio cannot be used as a biological marker for obesity, diabetes and cardiovascular disease (Magne et al. 2020). It was reported that walnuts could increase the abundance of Firmicutes and reduced the abundance of Bacteriodetes (Byerley et al. 2017). Thus, the decrease of B/F ratio in The WG should not be considered as an adverse change in the intestinal microecological structure. At the genus level, the SCFA producing genera of unclassified_f_Lachnospiraceae, Blautia and especially the Roseburia increased notably in the WG group, compared with the group of NC. The butyrate-producing bacteria of unclassified_f_Lachnospiraceae was necessary for the health of colonic epithelial tissue and maturation of the immune system, which was also negatively correlated with the inflammatory bowel disease (IBD) (Steinmeyer et al. 2015; Sun et al. 2017). The lower-fat diet resulted in a significant increase in relative abundance of Blautia, while the higher-fat diet led to a significant decrease in Blautia. And the Blautia was negatively associated with the changes in serum total cholesterol (TC), low-density lipoprotein cholesterol (LDL-C) and non-high-density lipoprotein cholesterol (non-HDL-C) (Wan et al. 2019). Further, the Blautia was inversely associated with visceral fat accumulationin adults 20-76 years of age, both in males and females (Ozato et al. 2019). In summary, as one of the main SCFA producers Blautia showed notable negative correlation with obesity and insulin resistance and exerted anti-inflammatory effects (Benítez-Páez et al. 2020; Zhang et al. 2019b). Roseburia, producing short-chain fatty acids, especially butyrate, could sustain colonic motility, immunity maintenance and anti-inflammatory properties and the decrease in Roseburia abundance associated with several diseases (Tamanai-Shacoori et al. 2017). It was reported that the decrease in Roseburia abundance was positively correlated with IBD and the Roseburia abundance significantly increased in IBD-patients, after treatment (Imhann et al. 2018; Walujkar et al. 2018). The Roseburia abundance significantly increased in diabetic rats, after treatment of baicalein and Roseburia was notably positively correlated with SCFAs content and negatively correlated with the levels of triglyceride (TG), TC, blood glucose, lipopolysaccharide (LPS) and inflammation (Zhang et al. 2018). The reduction of Roseburia abundance was also associated with chronic kidney disease progression and Roseburia was negatively associated with C-reactive protein in plasma (Jiang et al. 2016). Studies have shown that the composition of the gut microbiota could be influenced by dietary composition and animal-based diets and plant-based diets could shape different types of gut microbiota (Dahl et al. 2020; Jia et al. 2020). The plant-based diet appears to be beneficial for human health by 
promoting gut microbiota (Tomova et al. 2019). The animal-based diet increased the abundance of bile-tolerant microorganisms of Bacteroides and decreased the levels of Firmicutes, such as Roseburia and Lachnospiraceae (David et al. 2014; Barrett et al. 2018), which were inherently consistent with the reduced B/F ratio of plant-derived WG. In summery, the gut microbiota of mice could be improved by WG, by the way of enhancing the levels of SCFAs producing bacteria of Roseburia, unclassified_f_Lachnospiraceae and Blautia.

\section{Conclusion}

In summary, WG could be used as a long-term safe supplementary dietary protein and the safety of WG was verified by analysis of the hematological parameters and hematological biochemical parameters, after a WG administration of 30 days. The WG supplementation significantly increased the level of IL6 and decreased the levels of TNF- $a$ and IL-10 slightly, which indicated WG could enhance of the levels of activated T cells and monocytes and antiinflammatory ability. Further, the level of IgG increased significantly and the levels of the IgM and IgA decreased notably in the WG group, compared with the NC group, which illustrated that WG could improve immunity by promoting the differentiation and maturation process of B cells. The 16S rRNA sequencing showed that WG could improve the gut microbiota of the mice. At the phylum level, the Bacteroidetes were reduced and Firmicutes were increased, in the WG group, compared with the NC group. At the genus level, the SCFA producing genera of unclassified_f_Lachnospiraceae, Blautia and Roseburia increased remarkably. It is notable that the percentage of Roseburia increased more than threefold (NC $2.27 \%$, WG 9.19\%). And the level changes of cytokines and immunoglobulin were associated with the gut microbiota.

\section{Declarations}

\section{Acknowledgements}

The authors wish to acknowledge the Zhongyuan Scholars in Henan (No. 192101510004), Major Public Welfare Projects of Henan Province (201300110300), Strategic Consulting Research Project of Henan Institute of Chinese Engineering Development Strategies (NO. 2020HENZT13).

\section{Conflicts of interest/Competing interests}

There are no conflicts of interest to declare.

\section{Ethics approval}

Institutional review board.

\section{Consent to participate}

All authors agree to participate in this study.

\section{Consent for publication}

All authors have seen the manuscript and approved to submit the manuscript to World Journal of Microbiology and Biotechnology.

\section{Availability of data and material (data transparency)}

The raw sequences of 16S rRNA gene sequencing have been deposited in the NCBI Sequence Read Archive (SRA) database under Accession Number: (SAMN17192475,SAMN17192476,SAMN17192477, SAMN17192478,SAMN17192479, SAMN17192480,.SAMN17192481,SAMN17192482,SAMN17192483,SA SAMN17192485,SAMN17192486, SAMN17192487,SAMN17192488, SAMN17192489)

The web link to the datasets:

https://www.ncbi.nlm.nih.gov/biosample/17192475

https://www.ncbi.nlm.nih.gov/biosample/17192476

https://www.ncbi.nlm.nih.gov/biosample/17192477

https://www.ncbi.nlm.nih.gov/biosample/17192478

https://www.ncbi.nlm.nih.gov/biosample/17192479

https://www.ncbi.nlm.nih.gov/biosample/17192480

https://www.ncbi.nlm.nih.gov/biosample/17192481

https://www.ncbi.nlm.nih.gov/biosample/17192482

https://www.ncbi.nlm.nih.gov/biosample/17192483

https://www.ncbi.nlm.nih.gov/biosample/17192484 
https://www.ncbi.nlm.nih.gov/biosample/17192485

https://www.ncbi.nlm.nih.gov/biosample/17192486

https://www.ncbi.nlm.nih.gov/biosample/17192487

https://www.ncbi.nlm.nih.gov/biosample/17192488

https://www.ncbi.nlm.nih.gov/biosample/17192489

\section{Code availability}

Not applicable

\section{Authors' contributions}

Guanghai Yu : Analyzed data and wrote the paper

Xiaoguo Ji: Designed the study and Performed the research

Jihong Huang: Conceived of and designed study

Aimei Liao: Participating in analyzed data

Pan Long: Participating in analyzed data

Yinchen Hou: Participating in analyzed data

Ming Hui: Participating in designed study

Weiyun Guo : Participating in analyzed data

\section{References}

Anh, Thu, Dang, Benjamin, Marsland (2019) Microbes, metabolites, and the gut-lung axis. Mucosal Immunology 12:843-850. doi: 10.1038/s41385-019-0160-6

Barrett HL, Gomez-Arango LF, Wilkinson SA et al (2018) A Vegetarian Diet Is a Major Determinant of Gut Microbiota Composition in Early Pregnancy. Nutrients 10:890. doi: 10.3390/nu10070890

Benítez-Páez AA-O, Gómez Del Pugar EM, López-Almela I et al (2020) Depletion of Blautia Species in the Microbiota of Obese Children Relates to Intestinal Inflammation and Metabolic Phenotype Worsening. mSystems. 5:e00857-19. doi: 10.1128/mSystems.00857-19

Brandolini A, Hidalgo A (2012) Wheat germ: not only a by-product. Int J Food Sci Nutr 63 Suppl 1:71-4. doi: 10.3109/09637486.2011.633898

Byerley LO, Samuelson D, Blanchard E 4th et al (2017) Changes in the gut microbial communities following addition of walnuts to the diet. J Nutr Biochem 48:94-102. doi: 10.1016/j.jnutbio.2017.07.001

Castellanos JG, Longman RS (2020) Innate lymphoid cells link gut microbes with mucosal T cell immunity. Gut Microbes. 11:231-236. doi: $10.1080 / 19490976.2019 .1638725$

Chang Y, Chen Y, Zhou Q et al (2020) Short-chain fatty acids accompanying changes in the gut microbiome contribute to the development of hypertension in patients with preeclampsia. Clin Sci (Lond) 134:289-302. doi: 10.1042/CS20191253

Dahl WJ, Rivero Mendoza D, Lambert JM (2020) Diet, nutrients and the microbiome. Prog Mol Biol Transl Sci 171:237-263. doi:

10.1016/bs.pmbts.2020.04.006

Dai J, Yang X, Yuan Y et al (2020) Toxicity, gut microbiota and metabolome effects after copper exposure during early life in SD rats. Toxicology 433434:152395. doi: 10.1016/j.tox.2020.152395

David LA, Maurice CF, Carmody RN et al (2014) Diet rapidly and reproducibly alters the human gut microbiome. Nature 505:559-63. doi: 10.1038/nature12820

Decourt B, Lahiri DK, Sabbagh MN (2017) Targeting Tumor Necrosis Factor Alpha for Alzheimer's Disease. Curr Alzheimer Res 14:412-425. doi:

$10.2174 / 1567205013666160930110551$

Farsakoglu Y Palomino-Segura M, Latino I et al (2019) Influenza Vaccination Induces NK-Cell-Mediated Type-II IFN Response that Regulates Humoral Immunity in an IL-6-Dependent Manner. Cell Rep. 26:2307-2315.e5. doi: 10.1016/j.celrep.2019.01.104

Hegazy AN, West NR, Stubbington MJT et al (2017) Circulating and Tissue-Resident CD4(+) T Cells With Reactivity to Intestinal Microbiota Are Abundant in Healthy Individuals and Function Is Altered During Inflammation. Gastroenterology 153:1320-1337.e16. doi: 10.1053/j.gastro.2017.07.047 
Imhann F, Vich Vila A, Bonder MJ et al (2018) Interplay of host genetics and gut microbiota underlying the onset and clinical presentation of inflammatory bowel disease. Gut 67:108-119. doi: 10.1136/gutjnl-2016-312135

Ji XG, Huang JH, Hui M et al (2018) Proteomic Analysis and Immunoregulation Mechanism of Wheat Germ Globulin. Protein Pept Lett 24:1148-1165. doi: $10.2174 / 0929866524666171026123054$

Jia WA-O, Zhen J, Liu A et al (2020) Long-Term Vegan Meditation Improved Human Gut Microbiota. Evid Based Complement Alternat Med 2020:9517897. doi: $10.1155 / 2020 / 9517897$

Jiang S, Xie S, Lv D et al (2016) A reduction in the butyrate producing species Roseburia spp. and Faecalibacterium prausnitzii is associated with chronic kidney disease progression. Antonie Van Leeuwenhoek 109:1389-96. doi: 10.1007/s10482-016-0737-y

Kemanetzoglou E, Andreadou E (2017) CNS Demyelination with TNF-a Blockers. Curr Neurol Neurosci Rep 17:36. doi: 10.1007/s11910-017-0742-1

Magne F, Gotteland M, Gauthier L et al (2020) The Firmicutes/Bacteroidetes Ratio: A Relevant Marker of Gut Dysbiosis in Obese Patients? Nutrients $12: 1474$. doi: $10.3390 /$ nu12051474

Martin-Gallausiaux CA-O, Marinelli LA-O, Blottière HA-O et al (2020) SCFA: mechanisms and functional importance in the gut. Proc Nutr Soc 2:1-13. doi: $10.1017 /$ S0029665120006916

Martin-Subero Jl, Oakes CC (2018) Charting the dynamic epigenome during B-cell development. Semin Cancer Biol 51:139-148. doi:

10.1016/j.semcancer.2017.08.008

Matsumoto Y, Fujita T, Hirai I et al (2009) Immunosuppressive effect on T cell activation by interleukin-16- and interleukin-10-cDNA-double-transfected human squamous cell line. Burns 35:383-9. doi: 10.1016/j.burns.2008.06.017

Muhsin M, Ajendra J, Gentil K et al (2018) IL-6 is required for protective immune responses against early filarial infection. Int J Parasitol 48:925-935. doi: 10.1016/j.ijpara.2018.05.011

Neumann C, Scheffold A, Rutz S (2019) Functions and regulation of T cell-derived interleukin-10. Semin Immunol 44:101344. doi: 10.1016/j.smim.2019.101344

Ozato N, Saito S, Yamaguchi T et al (2019) Blautia genus associated with visceral fat accumulation in adults 20-76 years of age. NPJ Biofilms Microbiomes. 5:28. doi: 10.1038/s41522-019-0101-x

Sanna S, van Zuydam NR, Mahajan A et al (2019) Causal relationships among the gut microbiome, short-chain fatty acids and metabolic diseases Nature Genetics. Nat Genet 51:600-605. doi: 10.1038/s41588-019-0350-x

Shuo W囚Meiqin H, Xue Y et al (2018) Gut microbiota mediates the anti-obesity effect of calorie restriction in mice. Sci Rep 8:13037. doi: 10.1038/s41598-01831353-1

Sigvardsson M (2018) Molecular Regulation of Differentiation in Early B-Lymphocyte Development. Int J Mol Sci 19:1928. doi: 10.3390/ijms19071928

Steinmeyer S, Lee K, Jayaraman A, Alaniz RC (2015) Microbiota metabolite regulation of host immune homeostasis: a mechanistic missing link. Curr Allergy Asthma Rep 15:24. doi: 10.1007/s11882-015-0524-2

Stilling RM, van de Wouw M, Clarke G et al (2018) The neuropharmacology of butyrate: The bread and butter of the microbiota-gut-brain axis? Neurochem Int 99:110-132. doi: 10.1016/j.neuint.2016.06.011

Sun M, Wu W, Liu Z, Cong Y (2017) Microbiota metabolite short chain fatty acids, GPCR, and inflammatory bowel diseases. J Gastroenterol 52:1-8. doi: 10.1007/s00535-016-1242-9Tamanai-Shacoori Z et al (2017) Roseburia spp.: a marker of health?

Tomova A, Bukovsky I, Rembert E et al (2019) The Effects of Vegetarian and Vegan Diets on Gut Microbiota. Front Nutr 6:47. doi: 10.3389/fnut.2019.00047

Vitetta L, Vitetta G, Hall S (2018) Immunological Tolerance and Function: Associations Between Intestinal Bacteria, Probiotics, Prebiotics, and Phages.Front Immunol 9:2240. doi: 10.3389/fimmu.2018.02240

Walujkar SA, Kumbhare SV, Marathe NP et al (2018) Molecular profiling of mucosal tissue associated microbiota in patients manifesting acute exacerbations and remission stage of ulcerative colitis. World J Microbiol Biotechnol 34:76 doi:10.1007/s11274-018-2449-0

Wan Y, Wang F, Yuan J et al (2019) Effects of dietary fat on gut microbiota and faecal metabolites, and their relationship with cardiometabolic risk factors: A 6-month randomised controlled-feeding trial. Gut 68:1417-1429. doi: 10.1136/gutjnl-2018-317609

Wang Y, Liu J, Burrows PD et al (2020) B Cell Development and Maturation. Adv Exp Med Biol 1254:1-22. doi: 10.1007/978-981-15-3532-1_1

Wu W, Zhang M, Ren Y et al (2017) Characterization and Immunomodulatory Activity of a Novel Peptide, ECFSTA, from Wheat Germ Globulin. J Agric Food Chem 65:5561-5569. doi: 10.1021/acs.jafc.7b01360 
Xie CB, Jane-Wit D, Pober JS (2020) Complement Membrane Attack Complex: New Roles, Mechanisms of Action, and Therapeutic Targets. Am J Pathol 190:1138-1150. doi: 10.1016/j.ajpath.2020.02.006

Xu Y, Ai C, Jiang P et al (2020) Oligosaccharides from Gracilaria lemaneiformis better attenuated high fat diet-induced metabolic syndrome by promoting the Bacteroidales proliferation. Food Funct 11:1049-1062. doi: 10.1039/c9fo01996k

Ząbczyńska M, Polak K, Kozłowska K et al (2020) The Contribution of IgG Glycosylation to Antibody-Dependent Cell-Mediated Cytotoxicity (ADCC) and Complement-Dependent Cytotoxicity (CDC) in Hashimoto's Thyroiditis: An in Vitro Model of Thyroid Autoimmunity. Biomolecules 10:171. doi: 10.3390/biom 10020171

Zhang BW, Sun WL, Yu N et al (2018) Anti-diabetic effect of baicalein is associated with the modulation of gut microbiota in streptozotocin and high-fat-diet induced diabetic rats. J Funct Foods 46:256-267. doi: org/10.1016/j.jff.2018.04.070

Zhang J, Wen C, Li C, Duan Y et al (2019a) Antioxidant Peptide Fractions Isolated from Wheat Germ Protein with Subcritical Water Extraction and Its Transport Across CacoCells. J Food Sci 84:2139-2146. doi: 10.1111/1750-3841.14720

Zhang L, Shi M, Ji J et al (2019b) Gut microbiota determines the prevention effects of Luffa cylindrica (L.) Roem supplementation against obesity and associated metabolic disorders induced by high-fat diet. FASEB J 33:10339-10352. doi: 10.1096/fj.201900488R

\section{Figures}

A

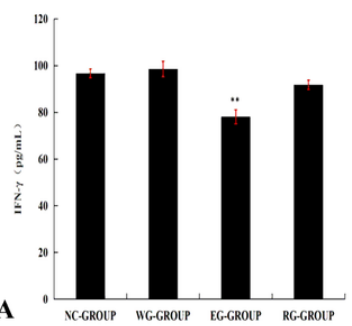

C
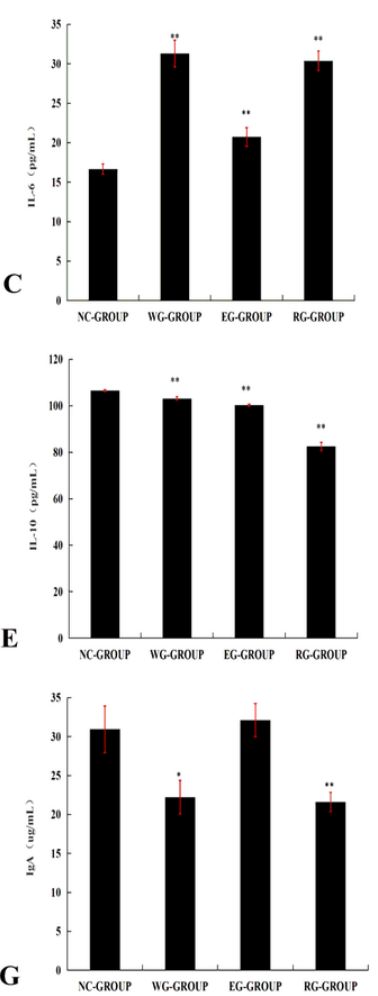

B

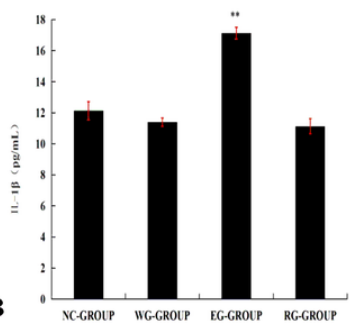

D
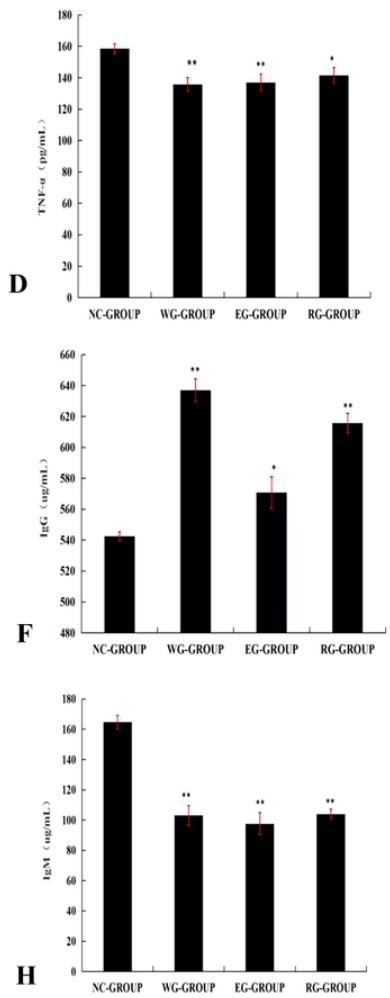

Figure 1

Plasma levels of various immune factors in different groups. 

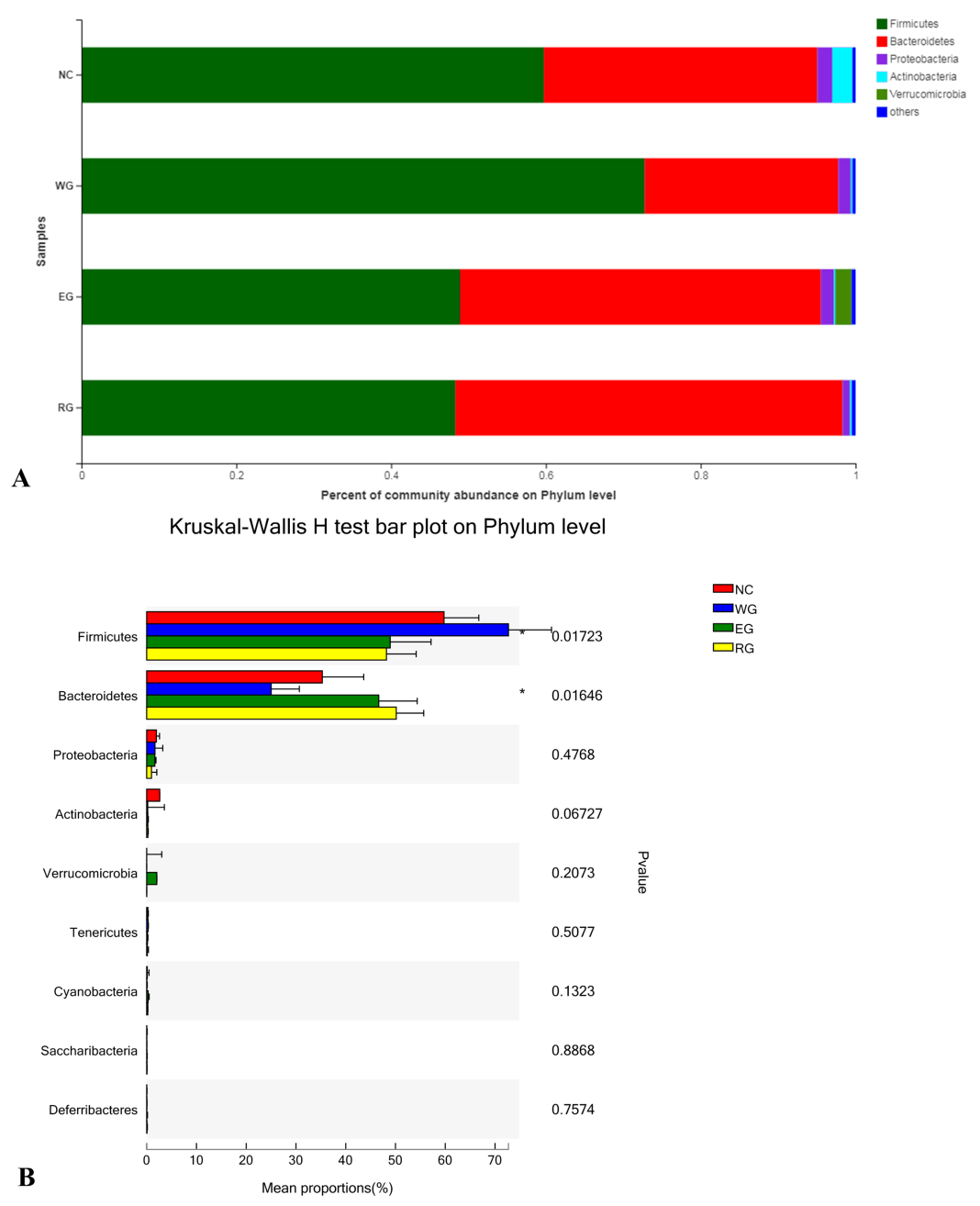

Figure 2

Community abundance on phylum level. (A) Microbial community bar plot at the phylum level with the relative abundance higher than $0.01 \%$. (B) KruskalWallis $\mathrm{H}$ test bar plot on phylum level.The asterisk represents significance $(*$ : $p<0.05)$. 


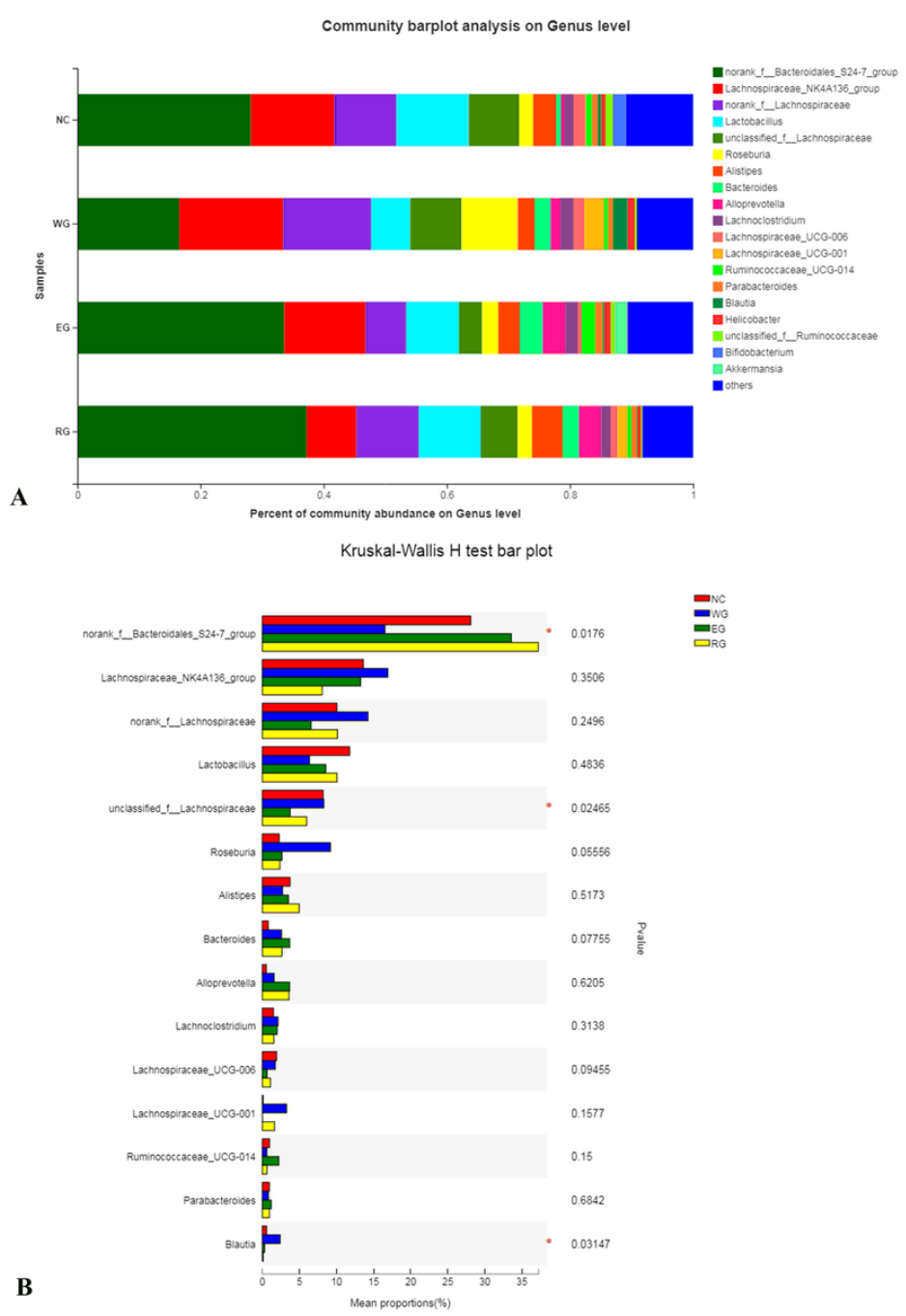

Figure 3

Community abundance on genus level. (A) Microbial community bar plot at the genus level with the relative abundance higher than $1 \%$. (B) Kruskal-Wallis $\mathrm{H}$ test bar plot on genus level. The asterisk represents significance $(*: p<0.05)$. 


\section{Student's t-test bar plot on Genus level}

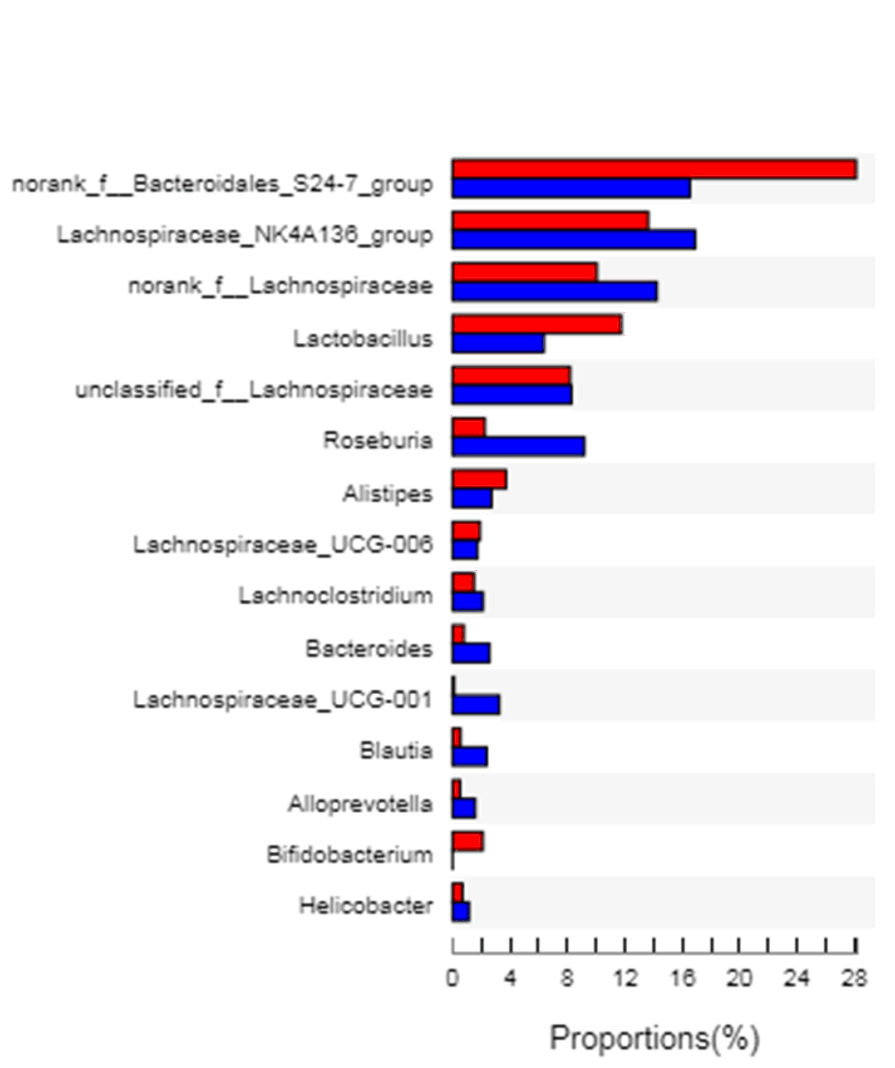

$95 \%$ confidence intervals

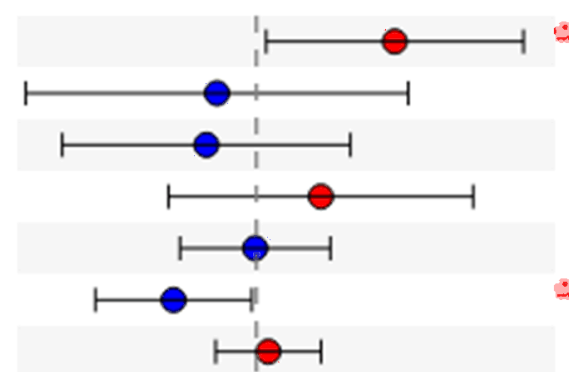

0.03945

0.6201

0.4128

0.326

0.8705

$\therefore \quad 0.04174$

0.5835

0.8517

0.5518

Figure 4

The microbiota composition difference between the groups of NC and WG. The asterisk represents significance $(*: p<0.05)$ 


\section{Spearman Correlation Heatmap}

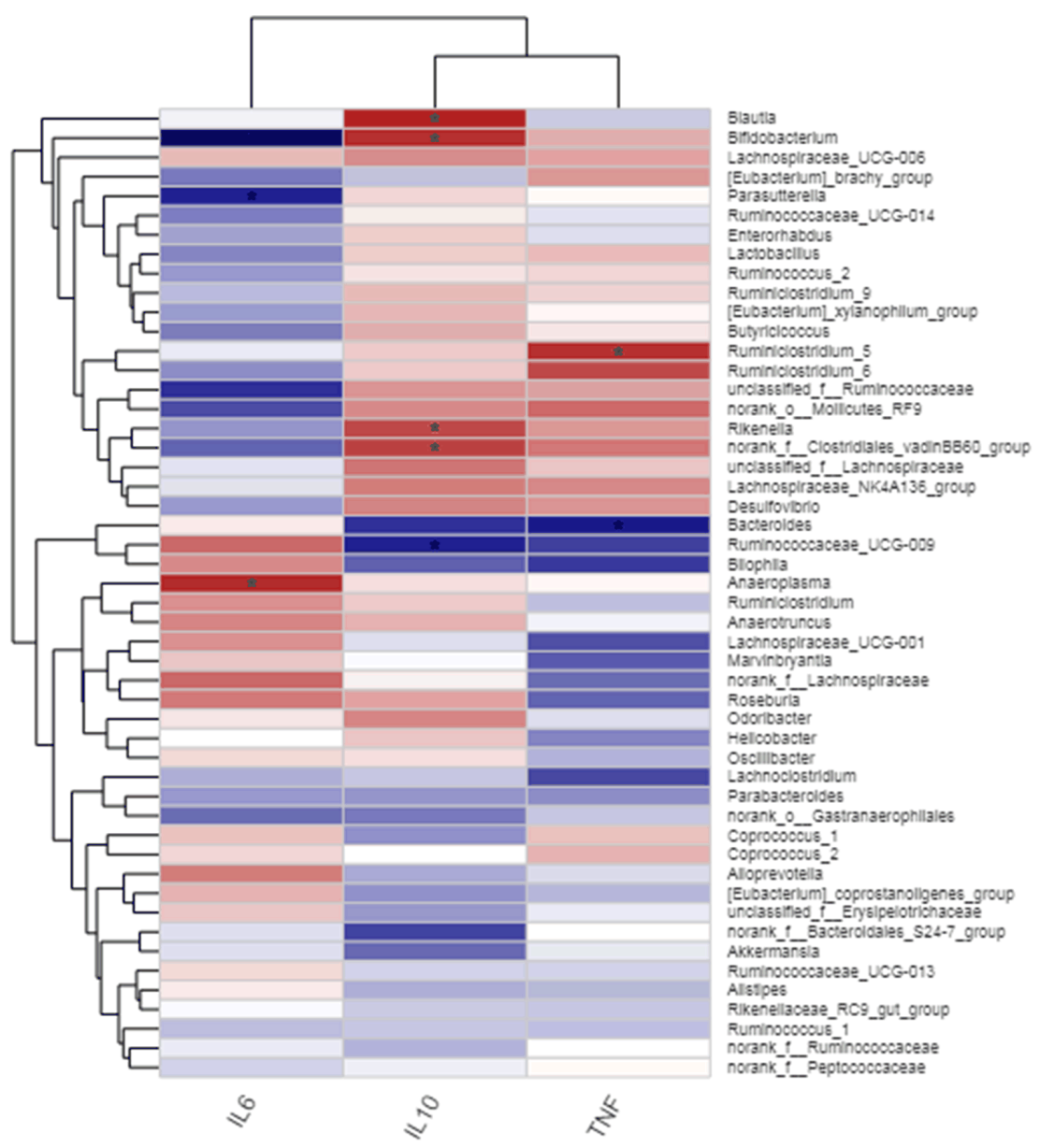

Figure 5

The correlation analysis between gut microbiota and cytokines. The asterisk represents significance $(*: p<0.05)$. 


\section{Spearman Correlation Heatmap}

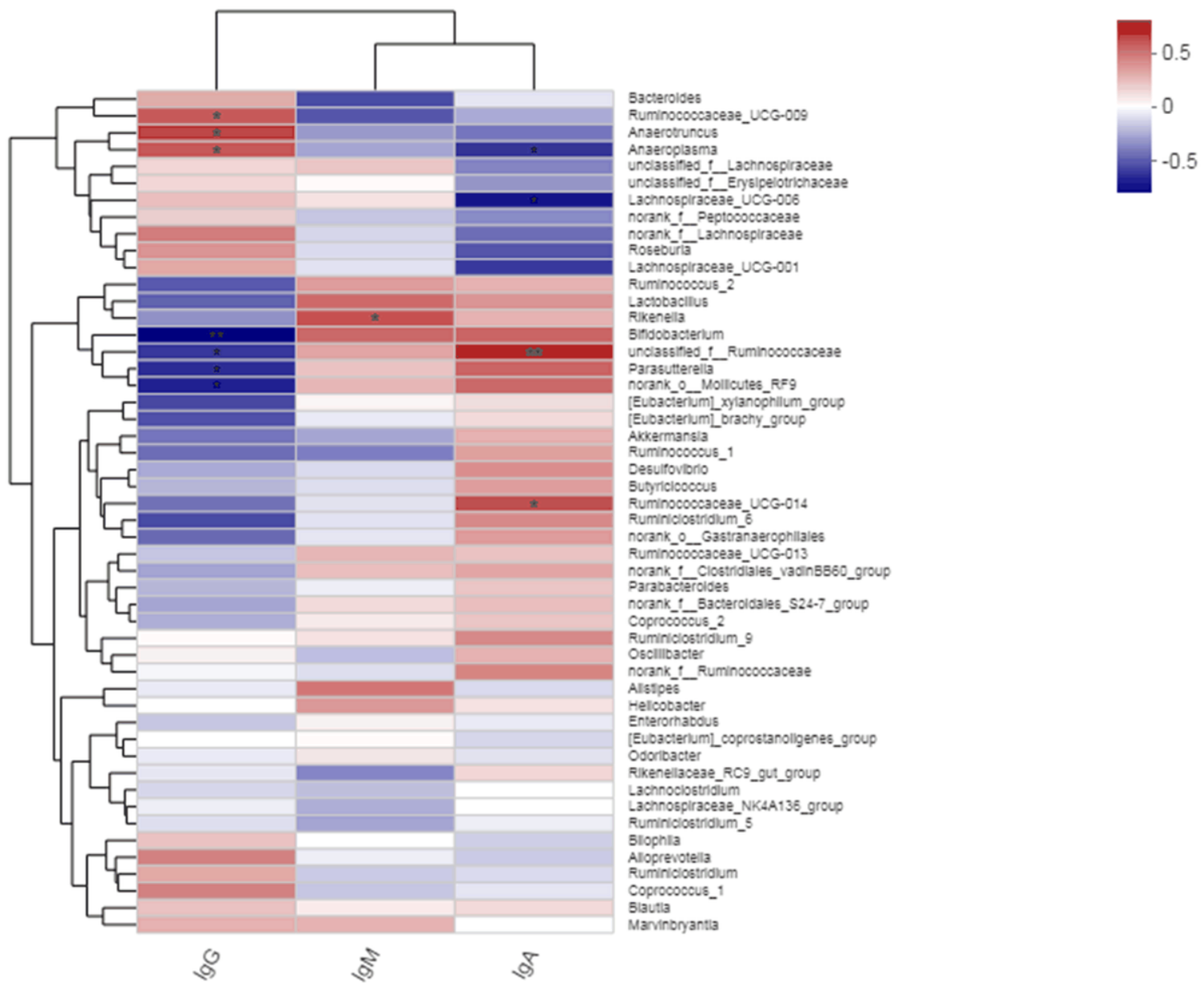

\section{Figure 6}

The correlation analysis between gut microbiota and immunoglobulin. The asterisk represents significance $(* *: p<0.01, *: p<0.05)$.

\section{Supplementary Files}

This is a list of supplementary files associated with this preprint. Click to download.

- GraphicalAbstracts.docx

- Authorchecklist.docx

- s1.png

- s2.png

- S3.png 